\title{
Evaluation of gas permeability in porous separators for polymer electrolyte fuel cells: CFD simulation based on micro X-ray computed tomography images
}

\author{
Soichiro Shimotori, ${ }^{1,}$ |* Toshihiro Kaneko, ${ }^{2}$ Yuta Yoshimoto, ${ }^{2}$ Ikuya \\ Kinefuchi, ${ }^{2}$ Amer Alizadeh, ${ }^{2}$ Wei-Lun $\mathrm{Hsu}^{2}{ }^{2}$ and Hirofumi Daiguji ${ }^{2}, \dagger$ \\ ${ }^{1}$ Toshiba Energy Systems \& Solutions Corporation, \\ 4-1 Ukishima-cho, Kawasaki-ku, Kawasaki 210-0862, Japan \\ ${ }^{2}$ Department of Mechanical Engineering, The University of Tokyo, \\ 7-3-1 Hongo, Bunkyo-ku, Tokyo 113-8656, Japan
}

(Dated: June 23, 2021)

\begin{abstract}
Pore structures and gas transport properties in porous separators for polymer electrolyte fuel cells are evaluated both experimentally and through simulations. In the experiments, the gas permeabilities of two porous samples, a conventional sample and one with low electrical resistivity, are measured by a capillary flow porometer, and the pore size distributions are evaluated with mercury porosimetry. Local pore structures are directly observed with micro X-ray computed tomography (CT). In the simulations, the effective diffusion coefficients of oxygen and the air permeability in porous samples are calculated using random walk Monte Carlo simulations and computational fluid dynamics (CFD) simulations, respectively, based on the X-ray CT images. The calculated porosities and air permeabilities of the porous samples are in good agreement with the experimental values. The simulation results also show that the in-plane permeability is twice the through-plane permeability in the conventional sample, whereas it is slightly higher in the low-resistivity sample. The results of this study show that CFD simulation based on micro X-ray CT images makes it possible to evaluate anisotropic gas permeabilities in anisotropic porous media.
\end{abstract}

\section{INTRODUCTION}

Fuel cells are promising electrochemical devices that use the reaction of hydrogen and oxygen to generate electrical power and are regarded as clean and low-cost power sources. These environmentally friendly chemical cells show a high energy conversion efficiency without the limitation imposed by the Carnot cycle. Owing to their excellent electrical conductivity and durability, polymer electrolyte fuel cells (PEFCs), which utilize a solid polymer thin membrane as electrolyte between the electrodes to prevent the leakage problems that occur in liquid electrolyte chemical cells, have attracted considerable attention over the past few years [1, 2. In general, PEFCs consist of a polymer membrane, catalyst layers, microporous layers, gas diffusion layers (GDLs), gas channels (GCs) and separators. Owing to the wide operating temperature range from room temperature to $100^{\circ} \mathrm{C}[3$, PEFCs can be used for a variety of applications, from large-scale facilities to portable systems such as engines for the next generation of electric vehicles, in which they are able to achieve a minimum response time for engine start-up and shutdown.

Water produced in PEFCs from the cathode reaction between protons and oxygen has a remarkable effect on the ion conductivity of the polymer electrolyte membrane and thus the performance of the cell. The water content of the membrane is subject to the surrounding vapor

\footnotetext{
* soichiro.shimotori@toshiba.co.jp Also at Department of Mechanical Engineering, The University of Tokyo.

† Corresponding author: daiguji@thml.t.u-tokyo.ac.jp
}

pressure and the water transport in the cell. In addition to water penetration from the cathode, water is electroosmotically transported from anode to cathode owing to the protons (positively charged) contained in the membrane [4. This gives rise to a dehydrated membrane, reducing the ion conductivity, and to an overhydrated cathode, hindering the oxygen supply to the cathode and thus resulting in a deterioration in cell efficiency. Therefore, to maximize the ion conductivity and performance of PEFCs, water management is an important task, but one that has yet to be optimized.

Humidification of the incoming reactant gases (external humidification) is one method to control the water content of PEFCs. To compensate for electro-osmotically transported water, humidification designs for the anode stream have been proposed [5]. Humidification of reactant gases at a temperature higher than the cell temperature has also been recommended [6]. In addition, the water balance across the cell and its sensitivity to changes in operating conditions have been studied [3, 7, 8]. Selfhumidification is another method to control the water content of PEFCs. This method employs a total heat and mass exchanger between the supply oxidant and the exhaust oxidant [9], which transfers vapor from exhaust to supply oxidant without any extra energy consumption. Yet another method for humidification is to supply water directly to the cell (internal humidification) by inserting a porous plate, which allows the supply of water from the coolant channels to be transferred to the anode without extra energy consumption [10, 11], as illustrated in Fig. 1(a).

As already mentioned, mitigation of the flooding phenomenon in an overhydrated cathode is also important to prevent deterioration in cell efficiency. One method 


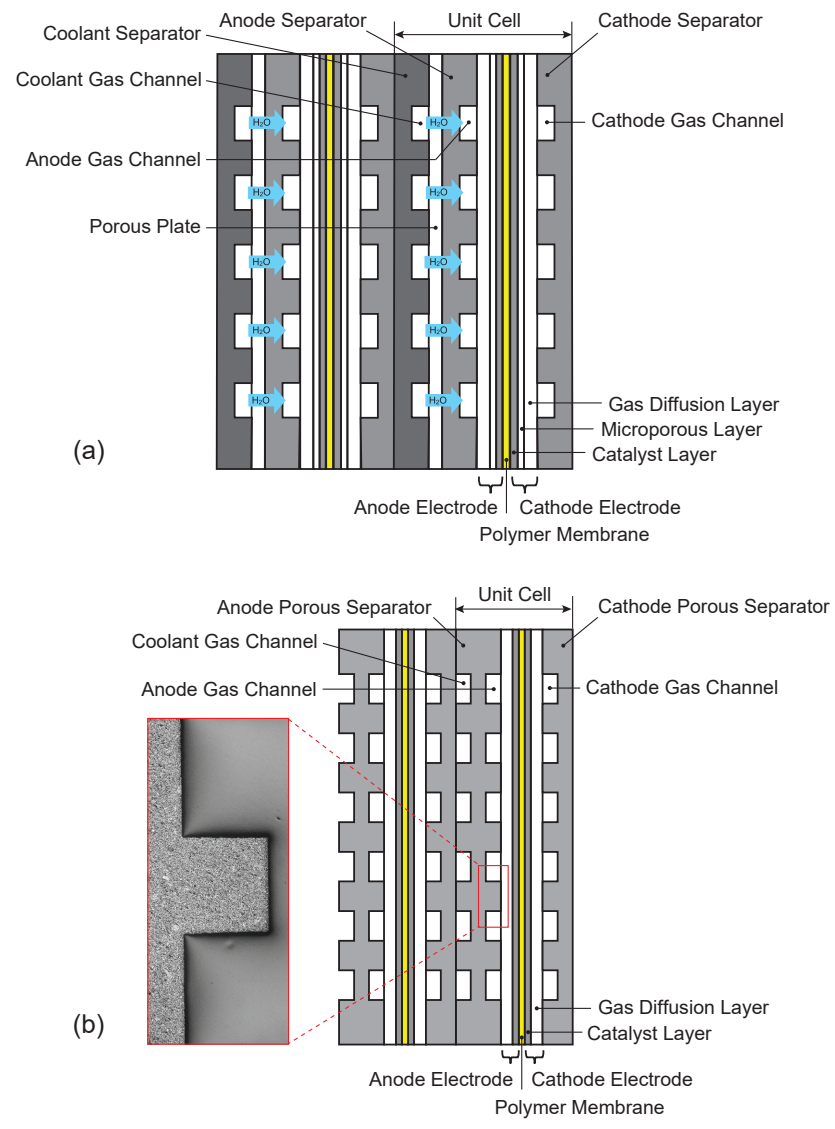

FIG. 1. (a) Schematics of (a) an internally humidified PEFC and (b) a PEFC with porous separators. The inset shows a scanning electron microscope image of a porous separator.

is to introduce porous separators at both cathode and anode, as illustrated in Fig. 1(b). As long as the pressure in the coolant channel is lower than that in the reactant gas channel, water can be continuously removed from the cathode through GDLs 12 14]. The incorporation of both an internal humidification unit and porous separators gives enhanced performance compared with conventional PEFCs using only external humidification, by providing a high water content in the polymer electrolyte membrane while removing a sufficient amount of liquid water from the cathode 15 .

In this context, the characteristics of water transfer across the porous separators becomes a key factor in the control of water supply and removal in PEFCs. It is important to enhance the water permeability while simultaneously maintaining a competitive electrical conductivity. However, the correlation between pore structure on the one hand and water permeability and electrical resistivity on the other has yet to be clarified. In general, the water permeability through a porous medium is defined by the product of the relative permeability of the liquid and the absolute permeability, where the relative permeability is a function of water saturation while the absolute permeability is an intrinsic property determined by the pore structure alone [16, 17. Therefore, to characterize water transport through a porous separator, evaluation of the absolute permeability of the porous medium is essential. Recently, the characterization of porous structures in PEFCs from the micro- to the nanoscale has been carried out using advanced tomographic techniques. For instance, catalyst layers have a pore structure of only a few tens of nanometers. Several studies have carried out three-dimensional (3D) transmission electron microscope (TEM) tomography to visualize the nanoscale structure [18]. Microporous layers have sub-micrometerscale and micrometer-scale pore structures. 3D scanning electron microscope (SEM) tomography has commonly been used to visualize the sub-micrometer-scale structure [19, 20]. On a larger scale of micrometers, 3D X-ray computed tomography (CT) has been employed [21, 22].

In the present study, 3D X-ray CT is performed to investigate the micrometer-scale pore structure of porous separators with different electrical resistivities. The scanned images of a 3D cylinder with diameter $1 \mathrm{~mm}$ and height $1 \mathrm{~mm}$ are binarized and the porosities of porous samples are evaluated. Then, the $3 \mathrm{D}$ cylindrical image is divided into cubes of side $200 \mu \mathrm{m}$ and the porosity of each cube is calculated. After porosity uniformity has been confirmed, the structural data for the central cube are transferred to voxel data for further calculations of transport properties. The pore size distribution for the central cube is confirmed to be the same to that measured with mercury porosimetry, and then effective diffusion coefficients of oxygen and air permeability are calculated with random walk Monte Carlo simulations and computational fluid dynamics (CFD) simulations, respectively. The objective of the former simulations is to evaluate the structural properties of porous separators such as anisotropy and tortuosity, while that of the latter simulations is to evaluate the anisotropic air transport properties in porous separators. The flow rates and permeabilities of air through porous samples calculated by CFD are compared with those measured by a capillary flow porometer. Under real operating conditions of PEFCs, water will be in both vapor and liquid states in the porous separator. The present study focuses on gas transport properties in a porous separator and clarifies the relationship between these properties and the porous structure. Analysis of the complex vapor-liquid two-phase flow in a porous separator is outside the scope of this study.

\section{EXPERIMENTAL METHODS}

\section{A. Sample preparation}

A porous separator for PEFCs was made from carbon. Such separators are usually made by pouring a mixture of carbon particles and resin into a patterned mold for a plate with small gas channels as shown in Fig. 11(b). However, in a patterned mold, the porous structure at 
TABLE I. Electrical resistivities and dimensions of porous samples.

\begin{tabular}{lcc}
\hline \hline & Sample A & Sample B \\
\hline Electrical resistivity $(\mathrm{m} \Omega \mathrm{m})$ & 0.356 & 0.249 \\
Dimensions (mm): & & \\
Flat porous separator & $320(L) \times 270(W) \times 2.5(T)$ \\
(a) Flow porometry & $30(D) \times 2.5(T)$ \\
(b) Mercury porosimetry & $50(L) \times 50(W) \times 2.5(T)$ \\
(c) X-ray CT & $10(L) \times 2(W) \times 2.5(T)$ \\
\hline \hline
\end{tabular}

the corners of the channels may differ from that on the flat surface of the channels, because the local stress acting on the mixture of carbon particles and resin depends on the local geometry. Thus, for the following experiments, a flat mold was used for the fabrication of a flat porous separator to ensure the uniformity of the porous structure.

Two types of flat porous separators with different electrical resistivities, a conventional type (sample A) and a low-resistivity type (sample B), were fabricated. To reduce electrical resistivity between carbon particles, carbon nanoparticles were added to sample B. In the fabrication process of a flat porous separator, the mixture of carbon particles and resin (plus carbon nanoparticles in sample B) was pored into a flat mold and was pressed in a certain direction. For the following experiments, the press direction is called through-plane direction and the normal to the press direction is called in-plane direction. Each of these two samples was cut into small pieces with three different dimensions for three different experiments: (a) flow porometry, (b) mercury porosimetry, and (c) Xray $\mathrm{CT}$. The electrical resistivity of each sample and the dimensions of the flat porous separators and small pieces for the three different experiments are shown in TableI. Note that the thickness direction $(T)$ is the through-plane direction, that is, the press direction and the length $(L)$, width $(W)$ and diameter $(D)$ directions are the in-plane directions.

\section{B. Permeability measurement}

The permeability of air through a porous sample in the thickness direction was measured by a capillary flow porometer (iPORE, Porous Materials Inc.) [23]. The disk-shaped sample was placed in a cylinder test rig, and air was supplied to the sample within the pressure range from 0 to $900 \mathrm{kPa}$ at room temperature. The air flow rate was measured by a mass flow meter in the air supply line. The permeability of air, $k$, can be calculated from the Darcy's law for gas flow with pressure-dependent gas density [24]:

$$
k=\frac{F}{A} \mu \frac{T}{\Delta P(1+\Delta P / 2 P)},
$$

where $F$ is the volumatic flow rate of air at the outlet, $A$ is the surface area of the sample, $\mu$ is the viscosity of air, $T$ is the thickness of the sample, $P$ is the outlet pressure which is kept at atomspheric pressure, and $\Delta P$ is the pressure difference between the inlet and the outlet.

\section{Pore size distribution measurement}

The pore size distribution of the porous samples was measured by a mercury porosimeter (AutoPore IV 9510, Micromeritics Inc.). The measured pore diameter ranged from $d_{p}=0.003$ to $100 \mu \mathrm{m}$. The contact angle and surface tension of mercury used in the calculation of pore diameter are $130^{\circ}$ and $485 \mathrm{dyn} / \mathrm{cm}$, respectively.

The $3 \mathrm{D}$ pore structure of a porous sample was repro-

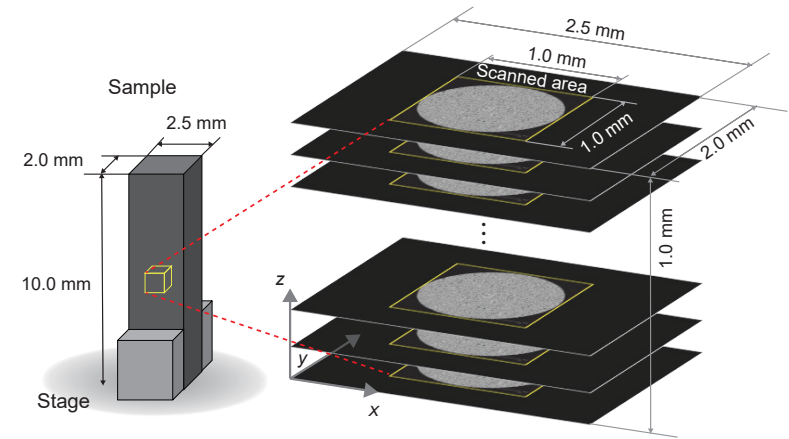

FIG. 2. Illustration of a porous sample on the stage and the cylindrical image scanned by X-ray CT.

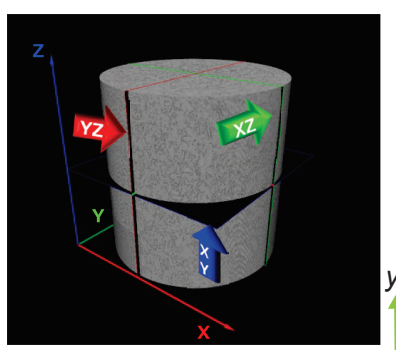

(a)

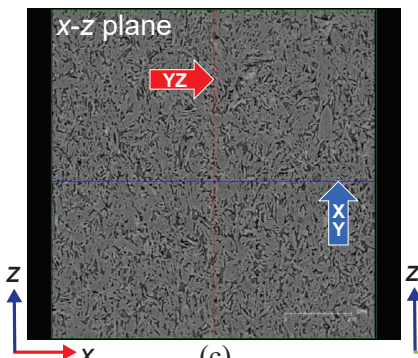

(c)

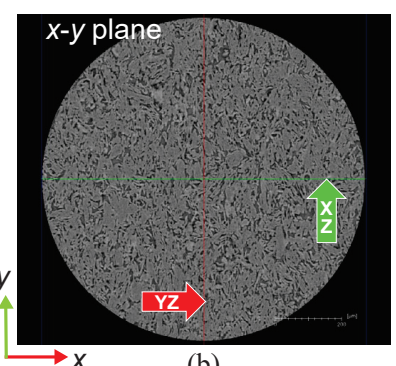

(b)

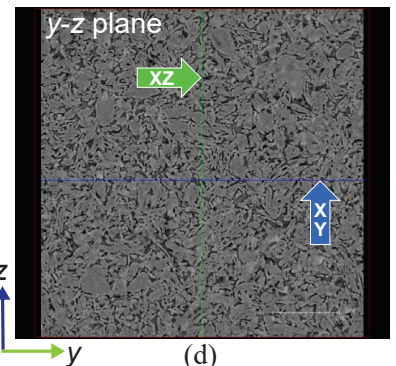

(d)
FIG. 3. (a) 3D image of a porous sample and its crosssectional images in (b) $x-y$, (c) $x-z$, and (d) $y-z$ planes (sample A). 
duced using micro X-ray CT and image processing. The rectangular sample was set on the stage of a micro X-ray CT scanner (ZEISS Xradia 520 Versa, Carl Zeiss Inc.), and $3 \mathrm{D}$ images were recorded by rotating the stage, as shown in Fig. 2, An X-ray source was operated at $5 \mathrm{~W}$ $(60 \mathrm{kV}$ and $0.83 \mathrm{~mA})$. The spatial resolution was $1 \mu \mathrm{m}$ per pixel for a $1 \mathrm{~mm} \times 1 \mathrm{~mm} \times 1 \mathrm{~mm}$ field of view. The central portion of the sample with dimensions of $10 \mathrm{~mm}(L)$ $\times 2 \mathrm{~mm}(W) \times 2.5 \mathrm{~mm}(T)$ was scanned, and the scanned data were used for image processing. Here, the $x, y$, and $z$ directions correspond to the thickness $T$, width $W$, and length $L$ of the sample, respectively. The scanned area in the $x-y$ plane was $1.0 \mathrm{~mm} \times 1.0 \mathrm{~mm}$. 995 cross-sectional images in the $x-y$ planes were stacked vertically in the $z$ direction. A 3D image of the sample in Cartesian coordinates, and the cross-sectional images in the $x-y, x-z$, and $y-z$ planes, are shown in Fig. 3. It should be noted that the $y-z$ plane corresponds to the in-plane of the sample (the $x$ direction corresponds to the thickness $T$ of the sample), while the $x-z$ and $x-y$ planes correspond to the through-plane of the sample (the $y$ and $z$ directions correspond to the width $W$ and length $L$ of the sample, respectively). In the scanned area, the high-brightness regions are the solid material consisting of carbon particles connected with resin, while the low-brightness regions are the pore regions. The remaining regions outside the circle provide no information. The pore structure data of a 3D cylindrical region thus obtained were used in the subsequent image processing

\section{X-ray computed tomography}

In the image processing, the images were binarized by using 'default' threshold in an image processing software ImageJ without considering any side information. The low-brightness regions below the threshold value were defined as pore regions, while the high-brightness regions above the threshold value were defined as solid regions. The number of pores and the volume of each pore were determined from the binarized image. The detected number of pores was 214514 . The pore with the largest volume was taken as an open pore, with the remaining pores being closed pores. Images of solid, void, open pore, and closed pores for the entire scanned area are shown in Fig. 4. The volumes of solid, void, open pore, and closed pores were $5.24 \times 10^{8}, 2.02 \times 10^{8}, 1.97 \times 10^{8}$, and $5.38 \times 10^{6} \mu^{3}$, respectively. The porosity $\epsilon$ is given by

$$
\epsilon=\frac{v_{p}}{v_{p}+v_{s}}
$$

where $v_{p}$ and $v_{s}$ are the pore and solid volumes, respectively.

\section{CALCULATION AND SIMULATION METHODS}

\section{A. Porosity distribution}

The following calculation and simulation were carried out using part of the binarized image, a cubic region of $200 \times 200 \times 200$ voxels extracted from a cylindrical scanned region as shown in Fig. 5. A square area of $600 \times 600$ pixels within the circular scanned area was divided into nine square areas of $200 \times 200$ pixels. In the $z$ direction, the scanned area was divided into five areas. A total of $45(=9 \times 5)$ cubic regions of $200 \times 200 \times 200$ voxels were thereby created in the entire scanned region as shown in Fig. 5. To evaluate the uniformity of the pore structure, the porosities of these 45 cubic regions were compared with each other. Each image was binarized at the same threshold level as mentioned in Sec. IID and a matrix of brightness data [either 1 (solid) or 0 (pore)] was created. The number of solid pixels was counted in each square area and then integrated over the 200 images in the $z$ direction

\section{B. Effective oxygen diffusion coefficient}

In order to evaluate the anisotropy and tortuosity of porous samples, the MSD calculation was performed in the Kn diffusion region where the trajectory of an oxygen molecule is easily obtained without considering collisions between oxygen molecules. The effective diffusion coeffi-

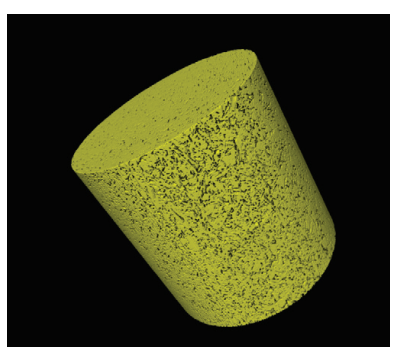

(a)

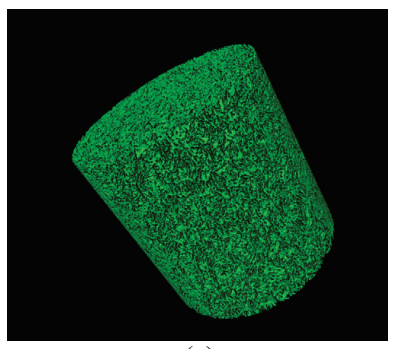

(c)

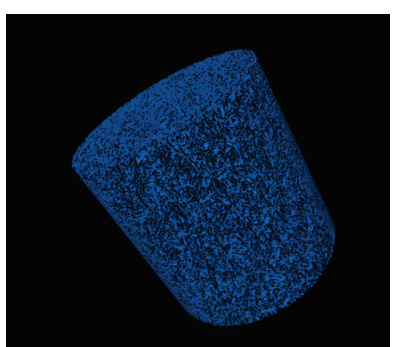

(b)

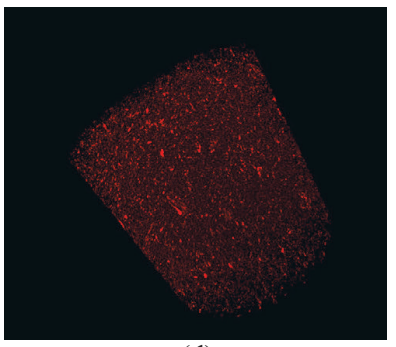

(d)
FIG. 4. Images of the entire cylindrical scanned region with $1 \mathrm{~mm}$ in diameter and $1 \mathrm{~mm}$ in height: (a) solid; (b) void; (c) open pore; (d) closed pores (sample A). 
cient for a porous structure, $D_{e}$, is given by [25 27]

$$
D_{e}=\frac{\epsilon D_{0}}{\tau}
$$

where $\epsilon$ is the porosity, $\tau$ is the tortuosity factor, which represents the tortuous nature of a porous medium, and $D_{0}$ is a reference diffusion coefficient, typically described by the Bosanquet equation [28]:

$$
D_{0}=\left(\frac{1}{D_{b}}+\frac{1}{D_{K}}\right)^{-1},
$$

where $D_{b}$ and $D_{K}$ are the diffusion coefficients in the bulk diffusion regime and the Knudsen diffusion regime, respectively. $D_{b}$ and $D_{K}$ are defined using the mean free path $\lambda$, the characteristic length $d$, and the mean molecular velocity $v$ as follows:

$$
\begin{gathered}
D_{b}=\frac{1}{3} \lambda v \\
D_{K}=\frac{1}{3} d v .
\end{gathered}
$$

By introducing the Knudsen number Kn, the following relation is satisfied:

$$
D_{b}=\operatorname{Kn} D_{K}
$$

where

$$
\mathrm{Kn}=\frac{\lambda}{d} .
$$

When $\mathrm{Kn} \rightarrow \infty$, the Bosanquet equation (4) becomes $D_{0} \approx D_{K}$, and when $\mathrm{Kn} \rightarrow 0$, it becomes $D_{0} \approx D_{b}$. The
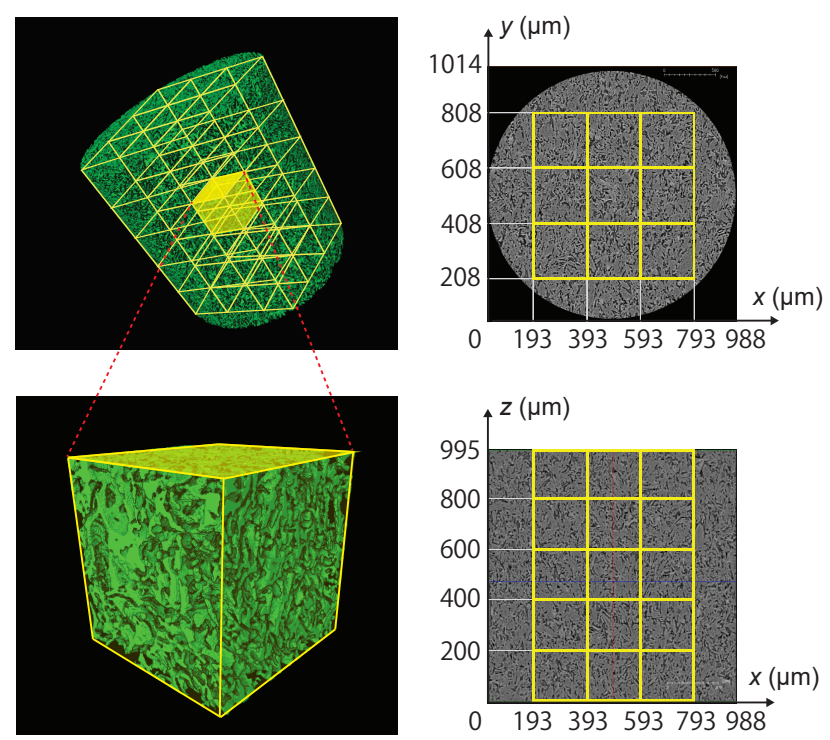

FIG. 5. Cubic analysis region of $200 \times 200 \times 200$ voxels extracted from a cylindrical scanned region with $1 \mathrm{~mm}$ in diameter and $1 \mathrm{~mm}$ in height. characteristic length $d$ was calculated using the following equation [16, 17]:

$$
d=\left(\frac{\left\langle l^{2}\right\rangle}{2\langle l\rangle^{2}}-\beta\right)\langle l\rangle,
$$

where $\langle l\rangle$ is the mean chord length and $\left\langle l^{2}\right\rangle$ is the mean squared chord length. $\left\langle l^{2}\right\rangle / 2\langle l\rangle^{2}$ expresses the correction for a non-exponential length distribution and is equal to 1 for an exponential distribution. $\beta$ is a constant related to the effect of redirecting collisions between walls and oxygen molecules [29, 30] and is given by

$$
\beta=-\sum_{m=1}^{\infty}\left\langle\cos \gamma_{m}\right\rangle,
$$

where $\gamma_{m}$ is the angle between the incident and reflected directions of oxygen molecules on the wall at the $m$ th collision. In the present study, the characteristic length $d$ was obtained as follows: 100000 lines were drawn randomly in a porous cube with a side of $200 \mu \mathrm{m}$, and the length of each line segment located in the pore region was calculated as the chord length $l$. Lines were drawn in both random directions for Monte Carlo simulation and Cartesian directions to avoid counting segments smaller than the pixel size. The analysis was performed by using a center cube located at $393 \mu \mathrm{m} \leq x \leq 592 \mu \mathrm{m}, 408 \mu \mathrm{m}$ $\leq y \leq 607 \mu \mathrm{m}$, and $400 \mu \mathrm{m} \leq z \leq 599 \mu \mathrm{m}$ (see Fig. 5).

The effective oxygen diffusion coefficient $D_{e}$ was calculated from the mean square displacement (MSD) as follows:

$$
D_{e}=\epsilon \lim _{t \rightarrow \infty} \frac{\left\langle|\mathbf{r}(t)-\mathbf{r}(0)|^{2}\right\rangle}{6 t},
$$

where $\mathbf{r}(t)$ is the position of an oxygen molecule at time $t$. It is noted that when $D_{e}$ in the $x$ direction is calculated, $\mathbf{r}(t)$ should be replated by $x(t)$ and $6 t$ should become $2 t$ in Eq. (11). The calculation was carried out with a random walk Monte Carlo simulation based on the mean-square displacement method [31, 32]. Specifically, 10000 oxygen molecules were inserted into the same porous cube of side $200 \mu \mathrm{m}$. The molecules were allowed to move in the pore region and reflect from the pore walls following the Knudsen cosine law. Mirror boundary conditions were applied on all the surfaces of the cubic cell. The velocity of oxygen molecules was set to $481.2 \mathrm{~m} / \mathrm{s}$, which is equivalent to the mean velocity at $350 \mathrm{~K}, \sqrt{8 k_{B} T / \pi m}$, where $k_{B}$ is Boltzmann's constant, $T$ is the temperature, and $m$ is the molecular mass of oxygen. The time step of the simulation was set to $5 \times 10^{-10} \mathrm{~s}$. In this calculation condition, the mean free path between molecular collisions for an oxygen molecule is much larger than the pore size because the number density of oxygen molecules in the pore region is extremely low. Therefore, Kn can effectively be assumed to be infinite and $D_{0}$ can be approximated as being the same as $D_{K}$. The value of $D_{K}$ was calculated from Eqs. (6), (9), and (10). The tortuosity factor $\tau$ was then obtained from Eqs. (3) and (11). 


\section{CFD simulation}

To calculate air permeability through the porous sample, a CFD simulation was performed using the X-ray CT images of porous cubes of sides 200 and $100 \mu \mathrm{m}$, respectively. The air flow was assumed to be compressible, single-phase, and laminar. The conservation equations for mass and momentum are

$$
\begin{aligned}
\nabla \cdot(\rho \mathbf{u}) & =0 \\
\rho(\mathbf{u} \cdot \nabla) \mathbf{u} & =-\nabla P+\mu \nabla^{2} \mathbf{u}
\end{aligned}
$$

where $\rho$ is the density of air, $\mathbf{u}$ is the velocity vector of air, $\mu$ is the viscosity of air, and $P$ is the pressure of air. $\rho$ was calculated by using the ideal gas law and the temperature was assumed to be $298 \mathrm{~K}$.

The volume mesh was created with the CT analyzer software Avizo. The center cube, located at $393 \mu \mathrm{m} \leq$ $x \leq 592 \mu \mathrm{m}, 408 \mu \mathrm{m} \leq y \leq 607 \mu \mathrm{m}$, and $400 \mu \mathrm{m} \leq$ $z \leq 599 \mu \mathrm{m}$, was extracted from the cylindrical scanned area as shown in Fig. 5. Figure 6 shows images of solid, void, open pore, and closed pores in the analysis region, corresponding to the images for the entire scanned region shown in Fig. 4. The volumes of solid, void, open pore, and closed pores are $5.66 \times 10^{6}, 2.21 \times 10^{6}, 2.13 \times 10^{6}$, and $8.10 \times 10^{4} \mu^{3}$, respectively. From the open pore data shown in Fig. 6(c), stereolithographic (STL) data were created as shown in Fig. 7(a). STL data were also created for a smaller cube of side $100 \mu \mathrm{m}$ as shown in Fig. 7(b). From these two sets of STL data, volume meshes with $33 \times 10^{6}$ and $7.3 \times 10^{6}$ cells were created as shown in Figs. 7(c) and 7(d), respectively. These volume meshes had seven interfaces: the six surfaces of the cube and the interface between void and solid inside the cube. With the air flow assumed to be in the $x$ direction, the inlet and outlet of the flow were on the two $y-z$ planes of the cube, and wall boundary conditions were applied to the other four surfaces of the cube. To calculate the flow rate at a fixed pressure drop, different constant pressures were applied on the inlet and outlet boundaries. The CFD simulations were performed for three flow directions $(x$, $y$, and $z$ ) with a Xeon Silver 4108 CPU and Star CCM+ v14.02.010-R8 software.

\section{RESULTS AND DISCUSSION}

\section{A. Measured air permeability, porosity and pore size distributions}

The air flow rate $F$ through the porous sample measured by a capillary flow porometer is shown in Fig. 8 (a). The measured value of $F$ increases proportionally to the pressure drop $\Delta P$ across the plate for both samples $\mathrm{A}$ and B. The permeability $k$ calculated using Eq. (1) is shown in Fig. 8(b). The values obtained are of the order of $10^{-14} \mathrm{~m}^{2}$. The probability density functions (PDFs) of pore diameter $d_{p}$ for samples $\mathrm{A}$ and $\mathrm{B}$ measured by

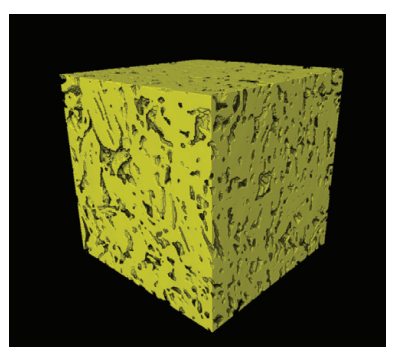

(a)

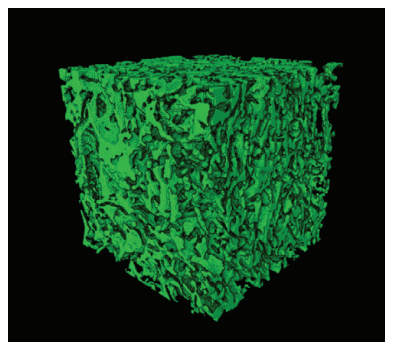

(c)

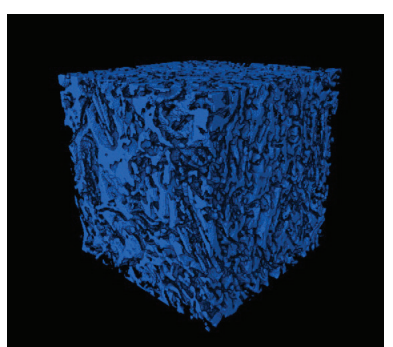

(b)

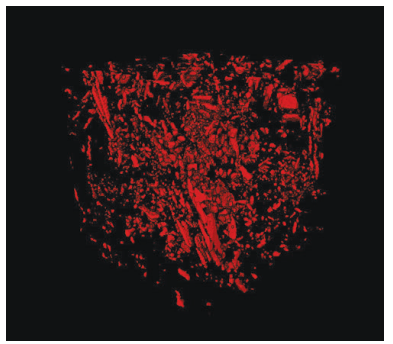

(d)
FIG. 6. Images of the cubic analysis region with the side of $200 \mu \mathrm{m}$ : (a) solid; (b) void; (c) open pore; (d) closed pores (sample A).

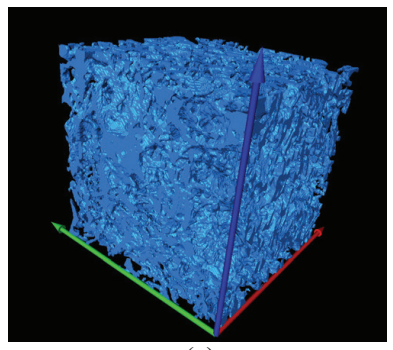

(a)

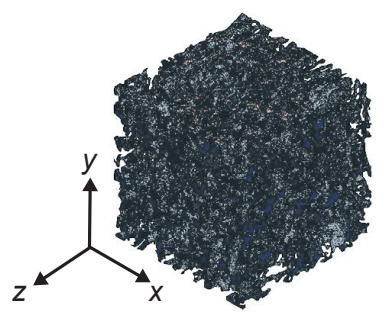

(c)

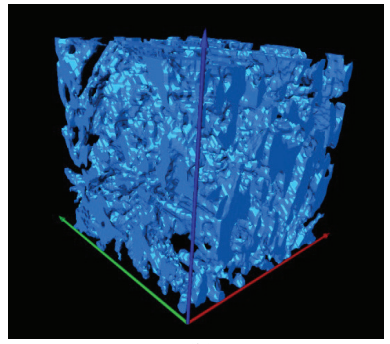

(b)

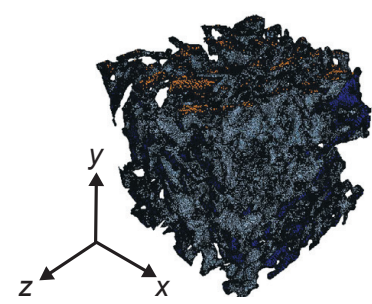

(d)
FIG. 7. STL data for cubic analysis regions with sides of (a) $200 \mu \mathrm{m}$ and (b) $100 \mu \mathrm{m}$, and volume meshes for cubic analysis regions with sides of (c) $200 \mu \mathrm{m}$ and (d) $100 \mu \mathrm{m}$ (sample A).

mercury porosimetry are shown in Fig. 9. Sample A has a sharp peak around $d_{p}=3.0 \mu \mathrm{m}$, while sample B has a peak around $d_{p}=2.6 \mu \mathrm{m}$ and a second, smaller, peak around $d_{p}=0.014 \mu \mathrm{m}$. Sample B includes carbon nanoparticles. The second peak around $d_{p}=0.014 \mu \mathrm{m}$ can be attributed to the nanopores formed by carbon nanoparticles. The measured pore volume and density 

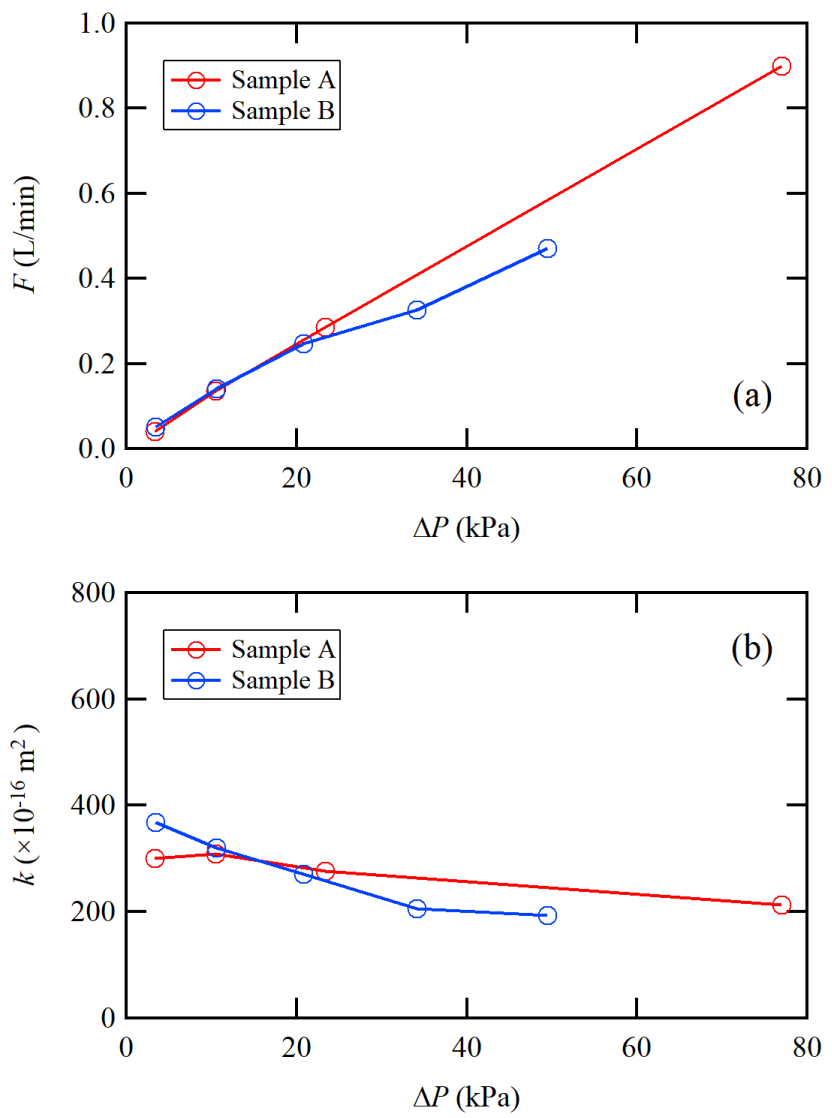

FIG. 8. (a) Air flow rate and (b) air permeability of samples $\mathrm{A}$ and $\mathrm{B}$ measured by a capillary flow porometer.

of sample A were $0.164 \mathrm{~cm}^{3} / \mathrm{g}$ and $1.51 \mathrm{~g} / \mathrm{cm}^{3}$, respectively, and thus the porosity was $0.164 \times 1.51=0.248$, while the measured pore volume and density of sample B were $0.138 \mathrm{~cm}^{3} / \mathrm{g}$ and $1.43 \mathrm{~g} / \mathrm{cm}^{3}$, giving a porosity of $0.138 \times 1.43=0.197$. The porosities of these two samples were also calculated using Eq. (2) from the images shown in Fig. 4. The results are summarized in Table III. The porosities obtained from the two different experimental approaches and image analyses have similar values, with the porosity of sample A being 5-7\% larger than that of sample B, which led to lower solid volume and higher electrical resistivity.

TABLE II. Pore volumes and porosities of samples A and B.

\begin{tabular}{|c|c|c|}
\hline & Sample A & Sample B \\
\hline & \multicolumn{2}{|c|}{ X-ray CT } \\
\hline Open pore volume $\left(\mu \mathrm{m}^{3}\right)$ & $1.97 \times 10^{8}$ & $1.54 \times 10^{8}$ \\
\hline Total volume $\left(\mu \mathrm{m}^{3}\right)$ & $7.27 \times 10^{8}$ & $7.27 \times 10^{8}$ \\
\hline \multirow[t]{2}{*}{ Porosity $\epsilon$} & 0.271 & 0.231 \\
\hline & \multicolumn{2}{|c|}{ Mercury porosimetry } \\
\hline Porosity $\epsilon$ & 0.248 & 0.197 \\
\hline
\end{tabular}

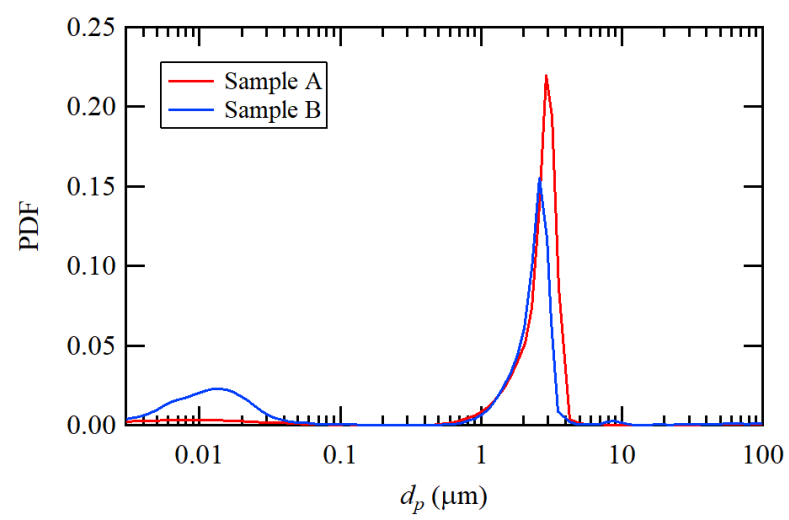

FIG. 9. Pore size distributions of samples A and B measured by mercury porosimetry: probability density functions (PDFs) of pore diameter $d_{p}$.

TABLE III. Chord lengths in random directions and other variables of samples $\mathrm{A}$ and $\mathrm{B}$.

\begin{tabular}{lcc}
\hline \hline & Sample A & Sample B \\
\hline$d(\mu \mathrm{m})$ & 2.46 & 2.25 \\
$\langle l\rangle(\mu \mathrm{m})$ & 3.65 & 3.26 \\
$\left\langle l^{2}\right\rangle\left(\mu \mathrm{m}^{2}\right)$ & 27.9 & 22.6 \\
$\left\langle l^{2}\right\rangle / 2\langle l\rangle^{2}$ & 1.05 & 1.06 \\
$\beta$ & 0.371 & 0.375 \\
\hline \hline
\end{tabular}

\section{B. Calculated pore size distribution and effective oxygen diffusion coefficient}

Figure 10(a) shows the porosity distribution of sample A along the $z$ direction calculated from the segmented data shown in Fig. 5 . From 45 segmented data $(3 \times 3 \times 5$ cubes in the $x, y$, and $z$ directions, respectively), the maximum and minimum porosities are 0.290 and 0.267 respectively, which correspond to $+4.2 \%$ and $-3.9 \%$ of the average porosity 0.278 . The porosity distribution of sample $\mathrm{B}$ along the $z$ direction was also calculated and is shown in Fig. 10(b). These results suggest that the porosity is almost uniform in the entire scanned region.

Figure 11 shows the distribution of the chord length $l$ in random directions of the two porous samples calculated using the method described in Sec. IIIB. Because discrete pixel data were used for the pore structure data, a spike appears every $1 \mu \mathrm{m}$. Figure 12 shows a comparison of the chord length distribution in Cartesian directions and the pore size distribution measured by mercury porosimetry. Although the chord length has discrete values, the peak values of the chord length and the measured pore diameter are around $3 \mu \mathrm{m}$. The calculated results for the chord lengths in random directions and other variables of the porous samples are summarized in Table III.

Figure 13 shows the MSDs of 10000 oxygen molecules in the porous structure calculated by Monte Carlo sim- 

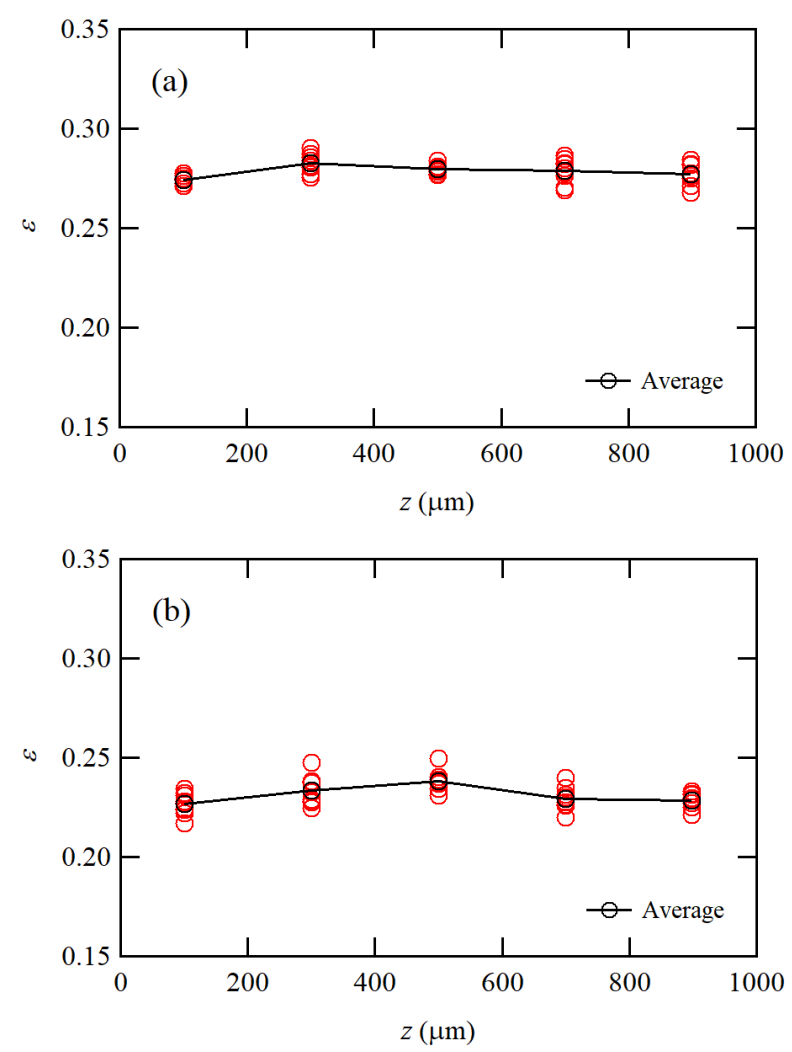

FIG. 10. Porosity distributions of (a) sample A and (b) sample B.

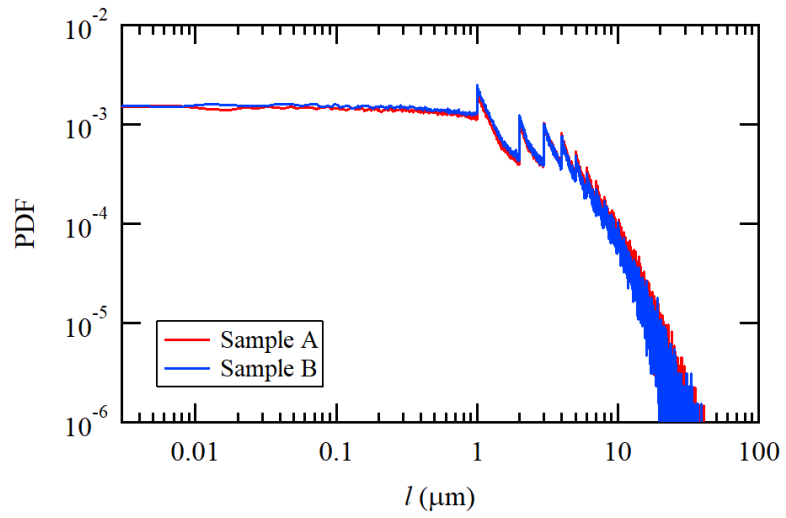

FIG. 11. Chord length distributions in random directions of samples A and B.

ulation. The insets shows the same graphs on a linear scale. The MSD increases steeply at $t<10^{-8} \mathrm{~s}$ because an oxygen molecule moves ballistically without colliding with the pore wall in this regime. The gradient of the MSD with respect to $t$ changes at around $t=10^{-8} \mathrm{~s}$. The MSD at $t=10^{-8} \mathrm{~s}$ is about $10^{-11} \mathrm{~m}^{2}$, which is of
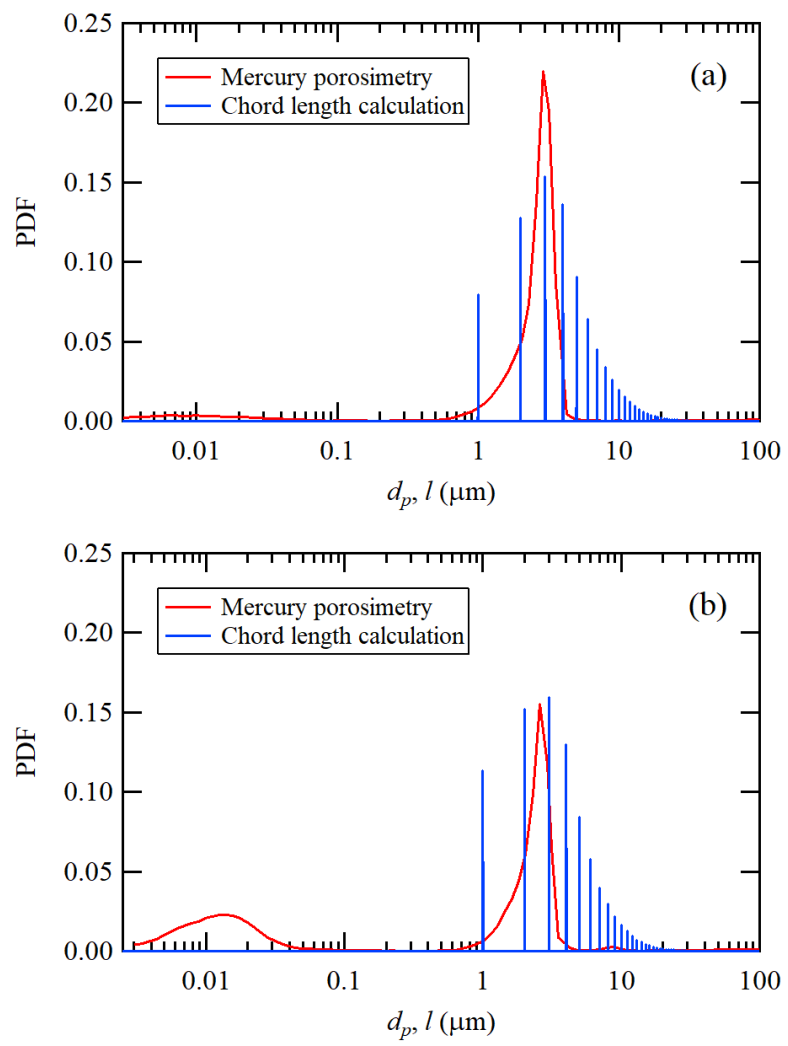

FIG. 12. Measured pore size distributions and calculated chord length distributions in Cartesian directions of (a) sample A and (b) sample B.

the same order of magnitude as the mean squared chord length $\left\langle l^{2}\right\rangle$. When $t$ is larger than $10^{-4} \mathrm{~s}$, the MSD is proportional to $t$, and the gradient gives the value of $D_{e} / \epsilon$ [see Eq. [11]]. Figure 13 also shows the $x, y$, and $z$ components of the MSDs. At $t>10^{-4} \mathrm{~s}$, in the case of sample A, the $x$ component of the MSD is clearly lower than the other two components [Fig. 13(a)], whereas for sample $\mathrm{B}$, the $x$ component is slightly less than the other two components [Fig. 13(b)]. This indicates that the effective diffusion coefficient of oxygen in the $x$ direction (i.e., the through-plane direction) is lower than that in the $y$ and $z$ directions (i.e., the in-plane directions). In this calculation, the number density of oxygen molecules is extremely low, and the mean free path between molecular collisions for an oxygen molecule is much larger than the pore size. Therefore, it is expected that the effective diffusion coefficient of oxygen largely depends on the porous structure, and the tortuosity factor, which represents the tortuous nature of a porous medium, can be evaluated appropriately. The calculated tortuosity factors $\tau$ are 4.4 and 5.8 for samples A and B, respectively. These values are slightly higher than the reference values of a porous material with a similar porosity of around 0.3 21]. The calculated diffusion coefficients, porosities, and tortuosity 

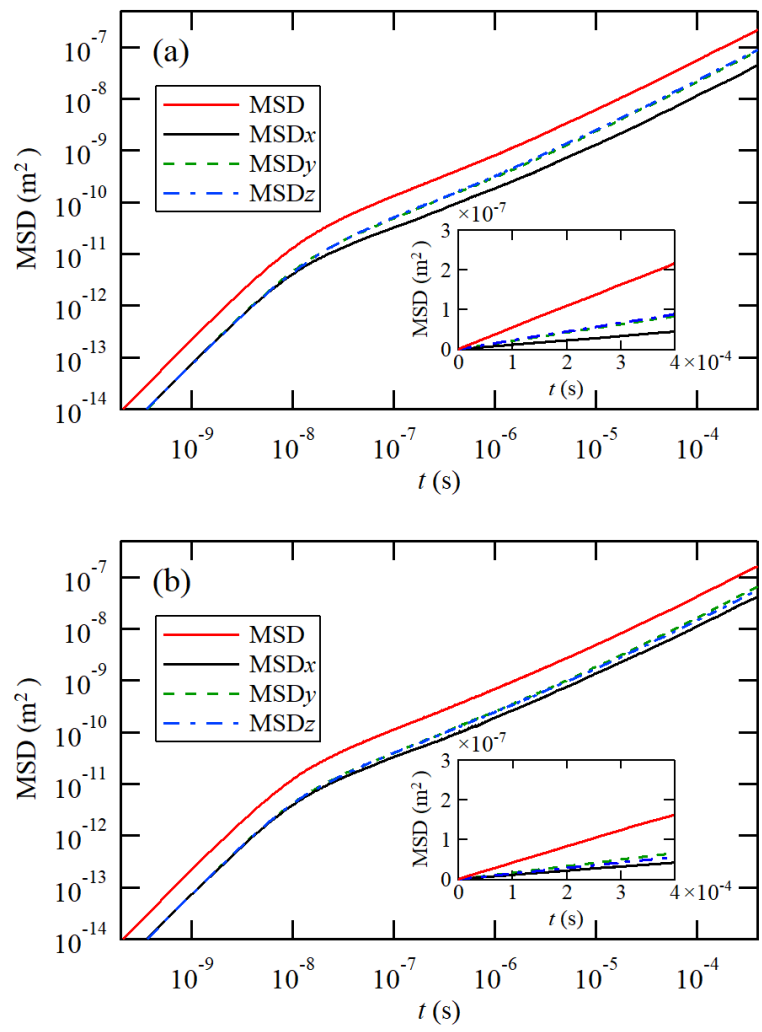

FIG. 13. Mean square displacements (MSDs), and their $x, y$, and $z$ components, of oxygen molecules in (a) sample A and (b) sample B. The insets show the same graphs on a linear scale.

TABLE IV. Oxygen diffusion coefficients and porosity and tortuosity factors of samples A and B.

\begin{tabular}{lcc}
\hline \hline & Sample A & Sample B \\
\hline$D_{e}\left(\mathrm{~m}^{2} / \mathrm{s}\right)$ & $2.52 \times 10^{-5}$ & $1.64 \times 10^{-5}$ \\
$D_{e}(x$ direction $)\left(\mathrm{m}^{2} / \mathrm{s}\right)$ & $1.56 \times 10^{-5}$ & $1.26 \times 10^{-5}$ \\
$D_{e}(y$ direction $)\left(\mathrm{m}^{2} / \mathrm{s}\right)$ & $2.93 \times 10^{-5}$ & $1.99 \times 10^{-5}$ \\
$D_{e}(z$ direction $)\left(\mathrm{m}^{2} / \mathrm{s}\right)$ & $3.08 \times 10^{-5}$ & $1.67 \times 10^{-5}$ \\
$D_{K}\left(\mathrm{~m}^{2} / \mathrm{s}\right)$ & $3.95 \times 10^{-4}$ & $3.60 \times 10^{-4}$ \\
$\epsilon$ & 0.281 & 0.240 \\
$\tau$ & 4.40 & 5.28 \\
\hline \hline
\end{tabular}

factors are summarized in Table IV.

\section{Calculated air permeability}

CFD simulations of air flow through sample A were performed with the volume mesh shown in Fig. 7(c). Figure 14 shows streamlines and pressure and flow velocity magnitude distributions when $\Delta P=40 \mathrm{kPa}$ was applied between two surfaces facing each other in the $y$ direction for the volume mesh of a cube of side $200 \mu \mathrm{m}$. The ve-

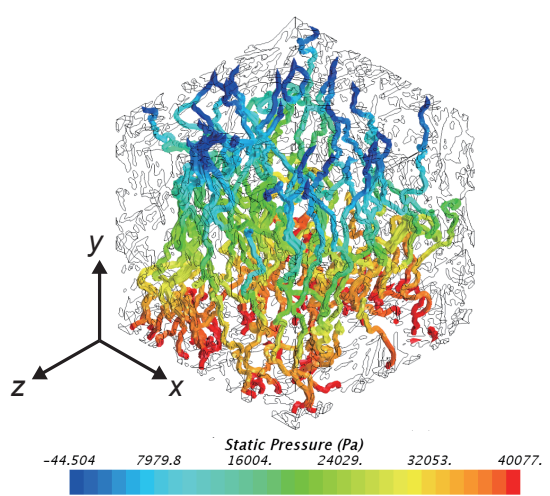

(a)

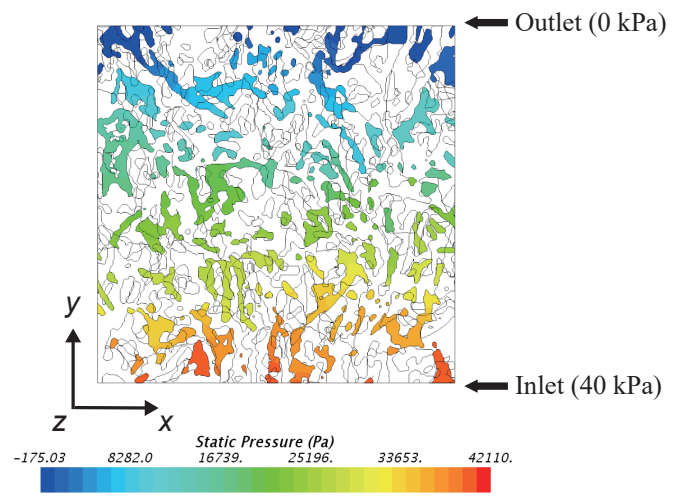

(b)

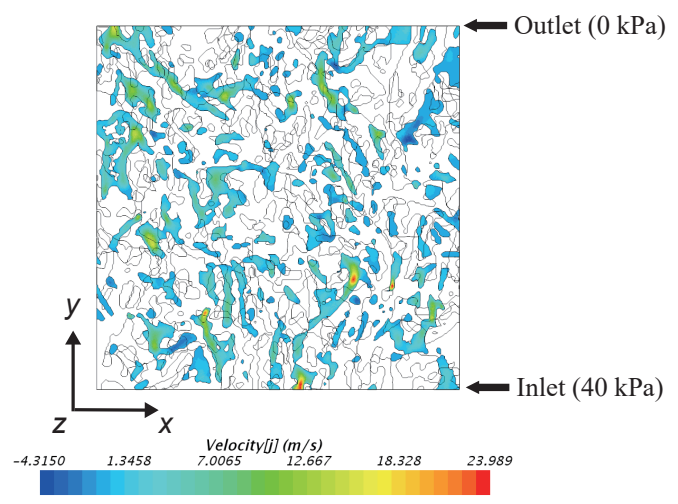

(c)

FIG. 14. (a) Streamlines, (b) pressure distribution, and (c) velocity magnitude distribution for the flow in the $y$ direction at $\Delta P=40 \mathrm{kPa}$ (sample $\mathrm{A}$ ).

locity magnitude distribution appears to be uniform, but the average velocity slightly increaces from the inlet to the outlet. The velocities at the inlet and the outlet are $1.8 \mathrm{~m} / \mathrm{s}$ and $2.2 \mathrm{~m} / \mathrm{s}$, respectively.

The volumetric flow rate and permeability [Eq. (1)] of sample A were calculated when $\Delta P=40 \mathrm{kPa}$ was applied in the $x, y$, and $z$ directions, respectively. The results are summarized in Table $\mathrm{V}$, The volumetric flow rate and permeability of sample $\mathrm{A}$ in the $x$ direction are about half of those in the $y$ and $z$ directions. In Sec. IVB, 
TABLE V. Volumetric flow rates and permeability of air in sample A obtained with cubic volume meshes of sides 100 and $200 \mu \mathrm{m}$ at $\Delta P=40 \mathrm{kPa}$.

\begin{tabular}{lccc}
\hline \hline & \multicolumn{3}{c}{$100 \times 100 \times 100 \mu^{3}$} \\
\cline { 2 - 4 } & \multicolumn{3}{c}{ Volumetric flow rates } \\
& Inlet $\left(\mathrm{m}^{3} / \mathrm{s}\right)$ & Outlet $\left(\mathrm{m}^{3} / \mathrm{s}\right)$ & Permeability $\left(\mathrm{m}^{2}\right)$ \\
\hline$x$ direction & $3.69 \times 10^{-9}$ & $4.90 \times 10^{-9}$ & $1.87 \times 10^{-14}$ \\
$y$ direction & $6.35 \times 10^{-9}$ & $8.26 \times 10^{-9}$ & $3.14 \times 10^{-14}$ \\
$z$ direction & $8.77 \times 10^{-9}$ & $1.17 \times 10^{-8}$ & $4.46 \times 10^{-14}$ \\
& \multicolumn{3}{c}{$200 \times 200 \times 200 \mathrm{\mu m}^{3}$} \\
\cline { 2 - 4 } & \multicolumn{3}{c}{ Volumetric flow rates } \\
& Inlet $\left(\mathrm{m}^{3} / \mathrm{s}\right)$ & Outlet $\left(\mathrm{m}^{3} / \mathrm{s}\right)$ & Permeability $\left(\mathrm{m}^{2}\right)$ \\
\hline$x$ direction & $8.88 \times 10^{-9}$ & $1.17 \times 10^{-8}$ & $2.24 \times 10^{-14}$ \\
$y$ direction & $1.67 \times 10^{-8}$ & $2.21 \times 10^{-8}$ & $4.19 \times 10^{-14}$ \\
$z$ direction & $1.81 \times 10^{-8}$ & $2.44 \times 10^{-8}$ & $4.65 \times 10^{-14}$ \\
\hline \hline
\end{tabular}

TABLE VI. Volumetric flow rates and permeability of air in samples $\mathrm{A}$ and $\mathrm{B}$ obtained with a cubic volume mesh of side $100 \mu \mathrm{m}$ at $\Delta P=20$ and $40 \mathrm{kPa}$.

\begin{tabular}{lccc}
\hline \hline & \multicolumn{3}{c}{ Sample A at $\Delta P=20 \mathrm{kPa}$} \\
\cline { 2 - 4 } & \multicolumn{3}{c}{ Volumetric flow rates } \\
& Inlet $\left(\mathrm{m}^{3} / \mathrm{s}\right)$ & Outlet $\left(\mathrm{m}^{3} / \mathrm{s}\right)$ & Permeability $\left(\mathrm{m}^{2}\right)$ \\
\hline$x$ direction & $2.01 \times 10^{-9}$ & $2.28 \times 10^{-9}$ & $1.89 \times 10^{-14}$ \\
$y$ direction & $3.50 \times 10^{-9}$ & $3.87 \times 10^{-9}$ & $3.21 \times 10^{-14}$ \\
$z$ direction & $4.81 \times 10^{-9}$ & $5.49 \times 10^{-9}$ & $4.55 \times 10^{-14}$ \\
& \multicolumn{4}{c}{ Sample B at $\Delta P=20 \mathrm{kPa}$} \\
\cline { 2 - 4 } & Volumetric flow rates \\
& Inlet $\left(\mathrm{m}^{3} / \mathrm{s}\right)$ & Outlet $\left(\mathrm{m}^{3} / \mathrm{s}\right)$ & Permeability $\left(\mathrm{m}^{2}\right)$ \\
\hline$x$ direction & $2.26 \times 10^{-9}$ & $2.50 \times 10^{-9}$ & $2.07 \times 10^{-14}$ \\
$y$ direction & $3.20 \times 10^{-9}$ & $3.60 \times 10^{-9}$ & $2.98 \times 10^{-14}$ \\
$z$ direction & $2.38 \times 10^{-9}$ & $2.71 \times 10^{-9}$ & $2.24 \times 10^{-14}$ \\
& \multicolumn{4}{c}{ Sample B at $\Delta P=40 \mathrm{kPa}$} \\
\cline { 2 - 4 } & Volumetric flow rates \\
& Inlet $\left(\mathrm{m}^{3} / \mathrm{s}\right)$ & Outlet $\left(\mathrm{m}^{3} / \mathrm{s}\right)$ & Permeability $\left(\mathrm{m}^{2}\right)$ \\
\hline$x$ direction & $4.13 \times 10^{-9}$ & $5.27 \times 10^{-9}$ & $2.00 \times 10^{-14}$ \\
$y$ direction & $5.84 \times 10^{-9}$ & $7.71 \times 10^{-9}$ & $2.93 \times 10^{-14}$ \\
$z$ direction & $4.36 \times 10^{-9}$ & $5.81 \times 10^{-9}$ & $2.21 \times 10^{-14}$ \\
\hline \hline
\end{tabular}

it was shown that the oxygen diffusivity of sample A in the $x$ direction was about half of that in the $y$ and $z$ directions. These anisotropic transport properties can be attributed to the anisotropic porous structure of sample A. Because the flat porous separator is made by pouring the mixture of carbon particles and resin into a mold and pressing in the $x$ direction, the porous structure should be compressed in the $x$ direction, and thus the transport in that direction should be suppressed. It is fair to say that the anisotropic transport properties have been successfully reproduced by the Monte Carlo calculation of oxygen diffusivity and the CFD calculation of air permeability based on the X-ray $\mathrm{CT}$ images of the porous structure.

Similar CFD simulations of air flow through sample A at $\Delta P=40 \mathrm{kPa}$ were performed with the cubic volume
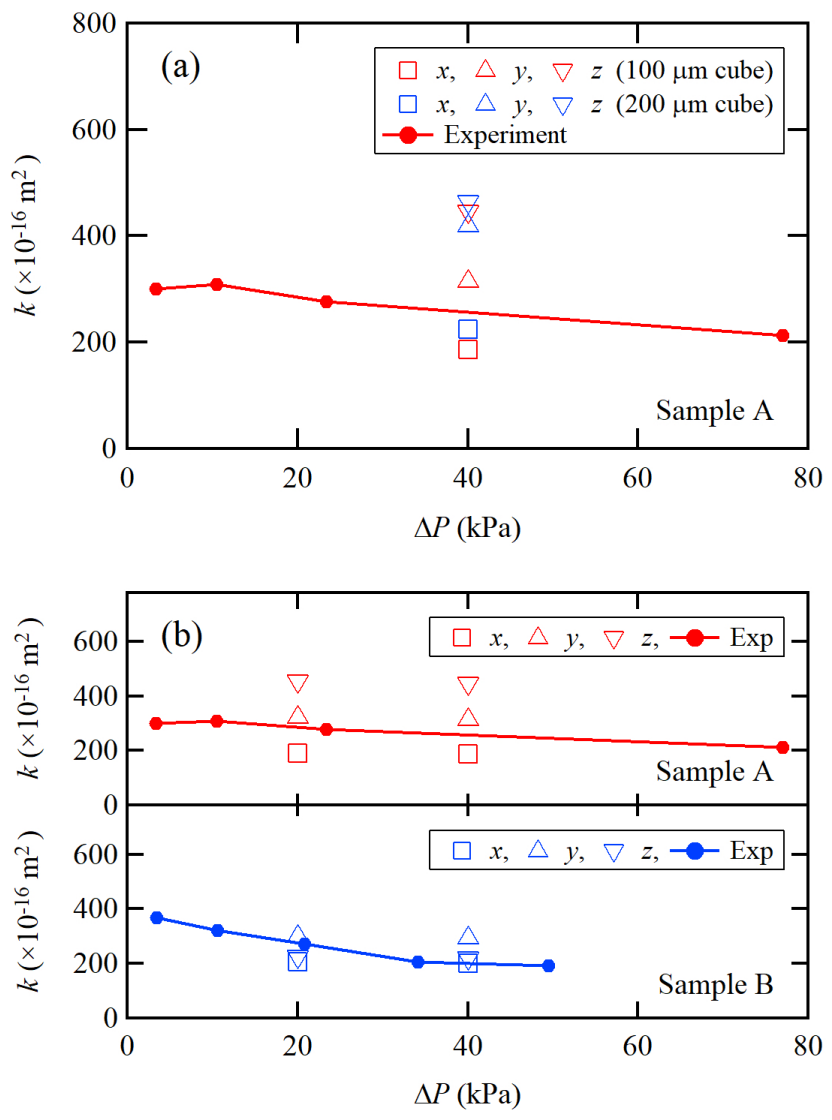

FIG. 15. Comparison between calculated and experimental results for air permeabilities in the $x, y$, and $z$ directions: (a) effect of analysis region size; (b) effect of sample type for a porous cube of side $100 \mu \mathrm{m}$.

mesh of side $100 \mu \mathrm{m}$ shown in Fig. 7(d). The calculated results for the volumetric flow rate and permeability of air in the $x, y$, and $z$ directions are shown in Table V. Figure 15(a) shows a comparison between the calculated air permeability for the two sizes of analysis region and the measured air permeability. All the values of air permeability are of the order of $10^{-14} \mathrm{~m}^{2}$. The calculated results for both sizes of analysis region show a similar trend, namely, that the volumetric flow rate and permeability in the $x$ direction are about half of those in the $y$ and $z$ directions.

Further simulations were performed using a cubic volume mesh of side $100 \mu \mathrm{m}$. The same CFD simulations of air flow through a porous sample were performed at $\Delta P=20 \mathrm{kPa}$ for sample $\mathrm{A}$ and at $\Delta P=20$ and $40 \mathrm{kPa}$ for sample B. The calculated results for the volumetric flow rate and permeability of air in the $x, y$ and $z$ directions are shown in Table VI In comparison between $\Delta P=20$ and $40 \mathrm{kPa}$ for each sample, the volumetric flow rate at $\Delta P=40 \mathrm{kPa}$ is about twice larger than that at $\Delta P=20 \mathrm{kPa}$ and the permeability in the $x, y$ and $z$ directions are almost the same both at $\Delta P=20$ and $40 \mathrm{kPa}$. Figure 15 (b) shows a comparison of permeabil- 
ity between samples $\mathrm{A}$ and $\mathrm{B}$, and it can be seen that the calculated permeability agrees with the experimental one. The permeability in the $x$ direction of sample $\mathrm{B}$ is close to that of sample $A$, while the porosity of sample $\mathrm{B}$ is lower than that of sample A, as shown in Table II. This suggests that the anisotropy of the porous structure in sample B is not as great as that in sample A. As already mentioned, sample B is a low electrical resistivity type. Because sample B includes carbon nanoparticles, the pore size distribution has a second, smaller, peak around $d_{p}=0.014 \mu \mathrm{m}$ (see Fig. 9). The carbon nanoparticles can affect the pore formation process during the solidification of carbon particles with a binder resin in a mold. The mechanism leading to the formation of an anisotropic pore structure is still to be determined, but the air permeability in the $y$ and $z$ directions was suppressed to the same degree as in the $x$ direction in sample B. The proposed method for evaluating gas permeability using CFD simulation based on micro X-ray CT images makes it possible to quantify gas permeability in a given direction inside an anisotropic porous medium.

\section{CONCLUSION}

A method to evaluate gas transport properties in porous structures has been proposed. This method involves the following procedures: (1) observation of local pore structures with X-ray CT; (2) creation of a 3D porous structure from the CT images; (3) analysis of gas diffusion coefficients and gas permeability in porous structures using random walk Monte Carlo simulations and CFD simulations, respectively. To validate the calculated pore size distribution and gas permeability, these values were also experimentally measured by mercury porosimetry and a capillary flow porometer, respectively. Specifically, 3D X-ray CT was used to observe the internal structure of porous PEFC separators of different electrical resistivities with a spatial resolution of $1 \mu \mathrm{m}$ per pixel over a cylindrical region of diameter $1 \mathrm{~mm}$ and height $1 \mathrm{~mm}$. The pore structural data were extracted from binarized image files, and the porosity was compared with the measured value. To evaluate the porous structure, the chord length distribution was calculated and compared with the pore size distribution obtained from mercury porosimetry. The effective oxygen diffusion coefficient in the pore structure was also calculated by random walk Monte Carlo simulations, and the difference between the in-plane and through-plane components was discussed. Finally, CFD simulations of air flow through porous samples were performed, and the volumetric flow rate and permeability of air were compared with those obtained from flow porometry.

The following conclusions can be drawn from this study.

The porosity calculated from the X-ray CT images [Eq. (2)] was in good agreement with that measured by mercury porosimetry. A $600 \mu \mathrm{m} \times 600 \mu \mathrm{m} \times 1000 \mu \mathrm{m}$ rectangular region was divided into 45 cubic regions each of $200 \mu \mathrm{m} \times 200 \mu \mathrm{m} \times 200 \mu \mathrm{m}$, and the porosity distribution was evaluated. The porosity of each cubic region was within $\pm 4.2 \%$ of the average value. Although the chord length had discrete values, its peak value was in good agreement with the peak pore diameter of around $3 \mu \mathrm{m}$ measured by mercury porosimetry.

The MSD of oxygen in the $x$ direction (i.e., the through-plane direction) was clearly lower than those in the $y$ and $z$ directions (i.e., the in-plane directions) for sample A (the sample with conventional resistivity). The volumetric flow rate and permeability of air in the $x$ direction were also clearly lower than those in the $y$ and $z$ directions for sample A. These anisotropic transport properties can be attributed to the anisotropic porous structure of this sample. Because the flat porous separator was pressed in the $x$ direction during the solidification process of carbon particles with a binder resin in a mold, the porous structure should be compressed in that direction. Thus, anisotropic transport properties were successfully reproduced by Monte Carlo calculation of diffusivity and CFD calculation of permeability based on X-ray CT images of the porous structure.

The MSD of oxygen in the $x$ direction (i.e., the through-plane direction) was slightly lower than those in the $y$ and $z$ directions (i.e., the in-plane directions) for sample B (the low-resistivity sample). The air permeability in the $x$ direction of sample B was close to that of sample A, while the porosity of sample B was lower than that of sample A. These results suggest that sample B was solidified more isotropically and more tightly. As a result, the air permeabilities in the $x, y$, and $z$ directions were suppressed similarly. Thus proposed method using CFD simulation based on micro X-ray CT images makes it possible to evaluate anisotropic gas permeabilities in anisotropic porous media.
[1] T. E. Springer, T. A. Zawodzinski, and S. Gottesfeld, Polymer electrolyte fuel cell model, J. Electrochem. Soc. 138, 2334 (1991).

[2] A. Z. Weber and J. Newman, Modeling transport in polymer-electrolyte fuel cells, Chem. Rev. 104, 4679 (2004).

[3] D. M. Bernardi, Water-balance calculations for solidpolymer-electrolyte fuel cells, J. Electrochem. Soc. 137,
3344 (1990).

[4] T. A. Zawodzinski, J. Davey, J. Valerio, and S. Gottesfeld, The water content dependence of electro-osmotic drag in proton-conducting polymer electrolytes, Electrochimica Acta 40, 297 (1995).

[5] T. V. Nguyen and R. E. White, A water and heat management model for proton-exchange-membrane fuel cells, J. Electrochem. Soc. 140, 2178 (1993). 
[6] E. A. Ticianelli and 1. C. R. Derouin, Methods to advance technology of proton exchange membrane fuel cells, J. Electrochem. Soc. 135, 2209 (1988).

[7] D. P. Wilkinson, H. H. Voss, and K. Prater, Water management and stack design for solid polymer fuel cells, J. Power Sources 49, 117 (1994).

[8] T. F. Fuller and J. Newman, Water and thermal management in solid-polymer-electrolyte fuel cells, J. Electrochem. Soc. 140, 1218 (1993).

[9] S. Shimotori, K. Saito, and M. Hori, Development of anti-freeze PEFC stack for automobile applications, SAE Technical Paper 1999-01-2572 (1999).

[10] D. L. Wood, Y. S. Yi, and T. V. Nguyen, Effect of direct liquid water injection and interdigitated flow field on the performance of proton exchange membrane fuel cells, Electrochim. Acta 43, 3795 (1998).

[11] S. Shimotori and A. Sounai, Evaporative cooling in polymer electrolyte fuel cell, Denki Kagku 64, 706 (1996).

[12] R. Baldwin, M. Pham, A. Leonida, J. McElroy, and T. Nalette, Hydrogen-oxygen proton-exchange membrane fuel cells and electrolyzers, J. Power Sources 29, 399 (1990).

[13] R. Zaffou, J. S. Yi, H. R. Kunz, and J. M. Fenton, Temperature-driven water transport through membrane electrode assembly of proton exchange membrane fuel cells, Electrochem. Solid State Lett. 9, A418 (2006).

[14] J. S. Yi, J. D. Yang, and C. King, Water management along the flow channels of PEM fuel cells, AIChE J. 50, 2594 (2004).

[15] A. Z. Weber and R. M. Darling, Understanding porous water-transport plates in polymer-electrolyte fuel cells, J. Power Sources 168, 191 (2007).

[16] A. Z. Weber, R. M. Darling, and J. Newman, Modeling two-phase behavior in PEFC, J. Electrochem. Soc. 151, A1715 (2004).

[17] J. Bear, Dynamics of Fluids in Porous Media (Dover Publications, 1988).

[18] M. Okumura, Z. Noda, J. Matsuda, Y. Tachikawa, M. Nishihara, S. M. Lyth, A. Hayashi, and K. Sasaki, Correlating cathode microstructure with PEFC performance using FIB-SEM and TEM, J. Electrochem. Soc. 164, F928 (2017).

[19] D. Kanno, N. Shikazono, N. Takagi, K. Matsuzaki, and N. Kasagi, Evaluation of SOFC anode polarization simulation using three-dimensional microstructures reconstructed by FIB tomography, Electrochim. Acta 56, 4015 (2011).

[20] H. Iwai, N. Shikazono, T. Matsui, H. Teshima, M. Kishi- moto, R. Kishida, D. Hayashi, K. Matsuzaki, D. Kanno, M. Saito, H. Muroyama, K. Eguchi, N. Kasagi, and H. Yoshida, Quantification of SOFC anode microstructure based on dual beam FIB-SEM technique, J. Power Sources 195, 955 (2010).

[21] I. Kinefuchi, J. Oyama, K. Yokoyama, N. Kubo, T. Tokumasu, and Y. Matsumoto, Direct simulation Monte Carlo analysis of gas transport in microporous structure based on X-ray computed tomography, ECS Trans. 58, 71 (2013).

[22] K. N. Grew, Y. S. Chu, J. Yi, A. A. Peracchio, J. R. I. Jr., Y. Hwu, F. D. Carlo, and W. K. S. Chiu, Nondestructive nanoscale $3 \mathrm{D}$ elemental mapping and analysis of a solid oxide fuel cell anode, J. Electrochem. Soc. 157, B783 (2010).

[23] A. Jena and K. Gupta, An innovative technique for pore structure analysis of fuel cell and battery components using flow porometry, J. Power Sources 96, 214 (2001).

[24] F. A. L. Dullien, Porous Media: Fluid Transport and Pore Structure, 2nd ed. (Academic Press, 1992).

[25] N. Epstein, On tortuosity and the tortuosity factor in flow and diffusion through porous media, Chem. Eng. Sci. 44, 777 (1989).

[26] J. Zalc, S. C. Reyes, and E. Iglesia, The effects of diffusion mechanism and void structure on transport rates and tortuosity factors in complex porous structures, Chem. Eng. Sci. 59, 2947 (2004).

[27] A. Berson, H. W. Choi, and J. G. Pharoah, Determination of the effective gas diffusivity of a porous composite medium from the three-dimensional reconstruction of its microstructure, Phys. Rev. E 83, 026310 (2011).

[28] W. G. Pollard and R. D. Present, On gaseous selfdiffusion in long capillary tubes, Phys. Rev. 73, 762 (1948).

[29] P. N. Henrion, Knudsen diffusion in powders. Part 1 Critical examination of a gas diffusion relationship used in Knudsen flow permeametry, Powder Technol. 16, 159 (1977).

[30] B. Derjaguin, Flow of gases through porous media, C. R. Acad. Sci. URSS 53, 623 (1946).

[31] Y. Yoshimoto, T. Hori, I. Kinefuchi, and S. Takagi, Effect of capillary condensation on gas transport properties in porous media, Phys. Rev. E 96, 043112 (2017).

[32] T. Kaneko, Y. Yoshimoto, T. Hori, S. Takagi, J. Ooyama, T. Terao, and I. Kinefuchi, Relation between oxygen gas diffusivity and porous characteristics under capillary condensation of water in cathode catalyst layers of polymer electrolyte membrane fuel cells, Int. J. Heat Mass Transf. 150, 119277 (2020). 